\title{
All Optical Logical Operations Using Excitable Cavity Solitons
}

\author{
Adrian Jacobo, Damiá Gomila, Pere Colet and Manuel A. Matías \\ Instituto de Física Interdisciplinar y Sistemas Complejos, IFISC (CSIC-UIB), \\ Campus Universitat Illes Balears, E-07122 Palma de Mallorca, Spain
}

\begin{abstract}
We show theoretically that dissipative solitons arising in the transverse plane of nonlinear optical cavities show oscillatory and excitable regimes that can be used to perform all-optical logical operations. This allows for the construction of reconfigurable optical gates that can operate in parallel.

Optical computing, via photons instead of electrons, has long appealed researchers as a way of achieving ultrafast performance. Photons travel faster than electrons and do not radiate energy, even at fast frequencies. Despite the constant advances and miniaturization of electronic computers, optical computing remains a strongly studied subject. We suggest as a strategy to follow not just to seek to imitate electronic computers, but rather to try to fully utilize the intrinsic parallelism of these systems. Most of the systems studied in optical computing applications imply light propagation, for example optical correlators, already commercially used in optical processing applications [1]. Disipative Solitons [2] arising in nonlinear optical cavities, also known as Cavity Solitons (CS) [4], have been suggested as a potentially useful strategy for information storage where a bit of information os represented by a cavity soliton [3]. Beyond the stationary regime, CS can show oscillatory behavior and furthermore there is a route in which oscillating $\mathrm{CS}$ are destroyed, leading to an excitable regime [5]. Excitability has been found in a variety of systems [6] and is characterized by a nonlinear response under applied external perturbations. Only perturbations exceeding a certain threshold are able to elicit in the system a full and well defined response. Furthermore after one perturbation the system cannot be excited again within a refractory period of time. Excitability is behind excitation waves in heart tissue and the existence of action potentials in neurons. In this work we study the interaction of CS solitons in the oscillatory and excitable regimes and discuss the potential of cavity solitons, in particular in a Kerr medium, in carrying out computations, i.e., not just for information storage. The key is the excitability exhibited by these CS. In particular, we will show how to design logic gates with CS, namely the AND, OR and NOT gates, as by combination of them one can construct any logical operation.
\end{abstract}

We model the Kerr cavity using the Lugiato-Lefever equation [7],

$$
\frac{\partial E}{\partial t}=-(1+\imath \theta) E+\imath \nabla^{2} E+E_{i n}+\imath\left|E^{2}\right| E,
$$

where $E(\vec{x}, t)$ is the slowly varying amplitude of the electric field, $\vec{x}=(x, y)$ is the transverse plane and $\nabla^{2}=$ $\partial^{2} / \partial x^{2}+\partial^{2} / \partial y^{2}$. The first term in the rhs of Eq. (1) describes the losses and $E_{\text {in }}$ is the input field (driving). $\theta$ is a detuning between the cavity and $E_{i n}$, and the sign of the cubic term corresponds the so called self-focusing case.

If $E_{i n}=E_{0}$, where $E_{0}$ is an homogeneous field, Eq. (1) has a homogeneous steady state solution implicitly given by $E_{s}=E_{0} /\left(1+\left(\imath\left(\theta-I_{s}\right)\right)\right.$, where $I_{s}=\left|E_{s}\right|^{2}$. Depending on the values of $I_{s}$ and $\theta$ CS arise and can be stationary, oscillate in time while remaining stationary in space or display an excitable behavior $[5,8]$. In the last case if suitably perturbed (i.e. with a superthreshold perturbation) the system elicits an excitable excursion with the spatial shape of a CS before relaxing back to the steady state.

Oscillating CS are an example of non-punctual oscillators, i.e. oscillators with an internal structure. The interplay between the coupling and the internal structure of non-punctual oscillators is a general phenomenon not well understood, and this work aims to be an approach to the subject by means of the study of a prototypical case. Here we show how, when two oscillating CS are placed close together, the mutual interaction locks the CS to three different equilibrium distances and leads to the appearance of two limit cycles, one in phase and one in anti-phase. We also show that for very close distances between the structures a beating of the two frequencies of these new limit cycles is observed. Examples of this behaviors are illustrated in Fig. 1, where we show the maximum of each structure as a function of time, for different distances and values of the parameters.

As shown recently, pumping the system with a plane wave an a Gaussian beam on top of it, the threshold of excitability can be tuned [9]. Here we consider three excitable cavity soliton in a linear arrangement, whose position is fixed by the Gaussian localized beam. The two outer cavity solitons will represent the input signals, while the central one 
will be the output signal. By varying the excitability threshold and the distance between the structures the response of the central soliton to perturbations on the outer ones can be controlled and, therefore, different logic gates can be achieved. The logic gates reported in this work are two gates involving two inputs and one output (the AND and OR gates), and one gate involving one input and one output (the NOT gate). In Fig. 2 we show the operation of an OR gate when one inputs is 1 and the other ' 0 ' leading to a 1 bit at the output (center). In this gate, if both inputs are '1' the outpus is also '1'. By increasing the distance or the excitability threshold of the structures it is also possible to achieve an AND gate where both inputs are required to be '1' in order to produce a '1' at the output. The NOT gate can be also realized by using only one cavity soliton not in the excitable regime, but in the oscillatory one.

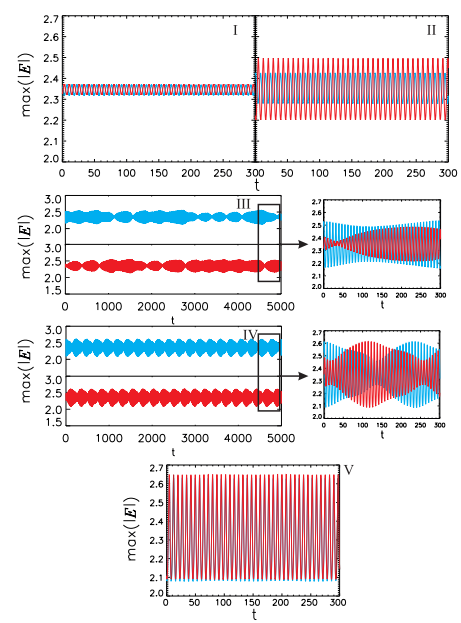

Figure 1: Oscillatory regimes for two coupled CS. Lines show the maximum value of one CS. I) Symmetric antiphase oscillations, II) Asymmetric antiphase oscillations III) Mixed mode beating IV) Phase locking V) In phase oscillations.
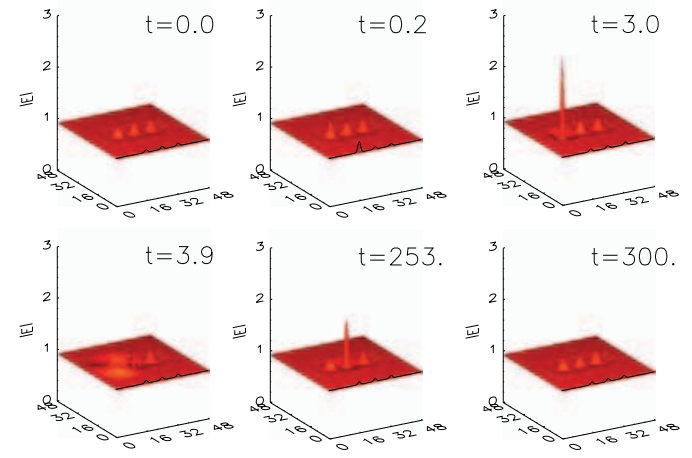

Figure 2: OR gate with input bits '1' (left) and '0' (right). This generates a '1' bit at the output (center).

In summary, oscillatory and excitable coupled CS show a rich variety of dynamical behaviors which can be used to perform all-optical logical operations, indicating that potential applications of optical cavity solitions can go way beyond optical storage.

Financial suport from MICINN (Spain) and FEDER (EU) through Grants No. FIS2007-60327 (FISICOS) and No. TEC2006-10009 (PhoDeCC) is acknowledged.

\section{References}

[1] E.J. Lerner, Optics do not always compute, Laser Focus World 36, 99 (2000)

[2] N. Akhmediev and A. Ankiewicz, eds. Dissipative Solitons: From Optics to Biology and Medicine, Lecture Notes in Physics 751 Springer, Berlin (2008).

[3] W.J. Firth and C.O. Weiss, Opt. Photonics News 13, 54 (2002).

[4] L.A. Lugiato ed., Feature section on cavity solitons, IEEE J. Quant. Elect., 39, \# 2 (2003).

[5] D. Gomila, M.A. Matías and P. Colet, Phys. Rev. Lett. 94, 063905 (2005).

[6] J. D. Murray, Mathematical Biology, 3rd Ed., Springer (2002).

[7] L. A. Lugiato and R. Lefever, Phys. Rev. Lett. 58, 2209 (1987).

[8] D. Gomila, A. Jacobo, M.A. Matías and P. Colet, Phys. Rev. E 75, 026217 (2007).

[9] A. Jacobo, D. Gomila, M.A. Matías and P. Colet, Phys. Rev. A 78, 053821 (2008). 